\title{
Tri-Band Microstrip Patch Antenna for Wireless Application
}

\author{
HALILU Adamu Jabire, Hong-xing Zheng * \\ Institute of Antenna and Microwave Techniques \\ Tianjin University of Technology and Education, Tianjin 300222, China \\ *Email: hxzheng@126.com
}

Keywords: Patch Antenna; Tri-band; Return Loss

\begin{abstract}
In order to implement the wireless devices with small size and good performance, this paper designs the microstrip patch antenna by using high frequency structure simulator software for wireless communications. The antenna works with triple bands in the range of $1.5 \mathrm{GHZ}$ to $4 \mathrm{GHZ}$ as the frequency sweep of the design. This design lays an emphasis on ensuring the antenna to work efficiently at 1.9GHZ, $2.45 \mathrm{GHZ}$ and $3.19 \mathrm{GHZ}$. These are very useful frequencies that can be used for varieties of wireless communications.
\end{abstract}

\section{Introduction}

The telecommunications does not stop to increase; it always tries to reach the best performances, the reliability and the efficiency with the lowest possible costs. In this domain, antenna establishes a basic element allowing the transmission of the electromagnetic waves in free space. Researchers find several types of antennas differing in cuts, geometrical shape and capacity of transmission [1]-[3]. The most serious limitation of the microstrip antenna is its narrow band, which is typically of the order of $1-5 \%$. However, the new generation of the communication, mobile or satellite communication provokes considerable changes in patch antenna, from which the various modern applications require a functioning in wide band and multiple bands. Novel investigation of a microstrip antenna compacts conception with a wide band, a triple frequency, a triple band, and an enhanced gain of operation were announced during the last years.

In this paper, researchers are interested in design of a microstrip antenna operating triple bands by using Ansoft High Frequency Structure Simulator (HFSS) software [4].

\section{Problem Description}

Patch antenna are inherently resonant antennas, being characterized by extremely low bandwidth. In addition, they are usually light in weight and easy to install because they are highly used in aircraft, satellite and missile applications [5]. Simplicity, low manufacturing cost and the flexibility to configure to specialized geometries are the advantages of patch antenna in the structure [6].

The fringing fields between the patch edge and the ground cause the microstrip patch antenna to radiate. A better performance in the antenna calls for a thick dielectric substrate having low dielectric constant, which provides better efficiency, larger bandwidth and better radiation. However, such a configuration results in large size of antenna. The design of a compact microstrip patch antenna demands higher dielectric constants, which are less efficient and result in narrower bandwidth. Therefore, an optimization is to be achieved between antenna dimensions and antenna performance, that is what happen to my design and the authors have to go for series of optimization before desired result used a higher dielectric constant. 
The microstrip patch antenna is designed to generate three resonant modes for operating at 1.9GHZ, 2.45GHZ and 3.19GHZ. Since the air-box, including a ground part, act as a primary source of radiation, the ground plate strongly influences the antenna performance, including return loss, bandwidth and radiation gain of operating frequencies [7]. The proposed patch antenna is very suitable for integration with personal communication services applications, wireless local area network applications and global positioning systems applications.

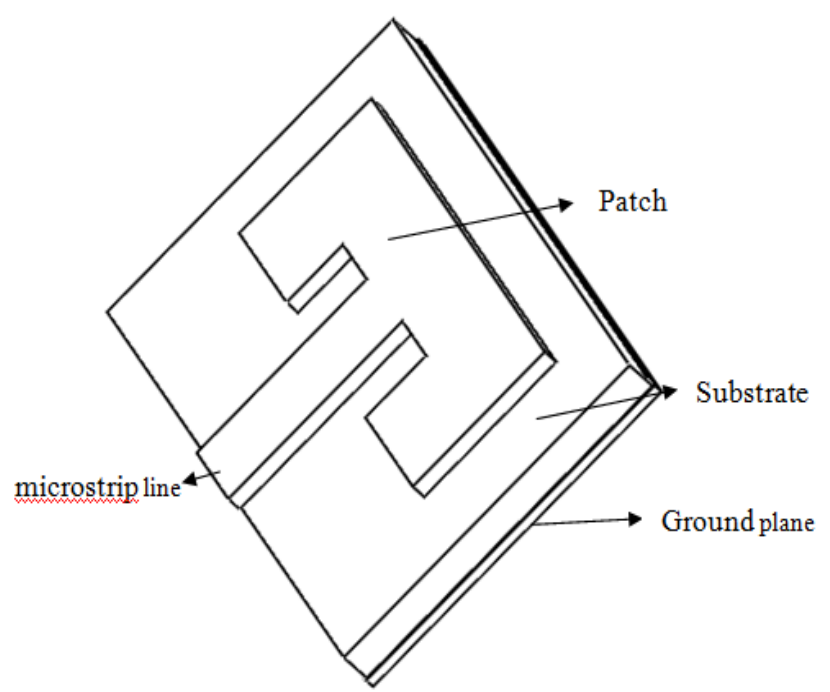

Fig. 1 Coaxial Feed/Probe Coupling

\section{Proposed Approach}

Fig. 1 shows the geometry of the proposed antenna with coaxial probe feed as the feeding type, which initially an inner feed conductor of the coaxial cable is connected at the centre of the patch being extruded to the substrate and ground plate. The geometrical parameters of the length and width of the patch are optimized in an attempt to achieve the designed goals at both the 1.9GHZ, 2.45GHZ and 3.19GHZ bands. The patch was designed as rectangular shaped resonating on fr4-epoxy substrate with dielectric constant of 4.4 and height of $1.6 \mathrm{~mm}$. The dimension of the antenna is $74.4 \mathrm{~mm} \times 55.98 \mathrm{~mm}$, i.e, $74.4 \mathrm{~mm}$ length (L) and width (W) of $55.98 \mathrm{~mm}$. At first, when the feeding point was at the centre, researchers got only one resonating frequency. However, when the feeding port was shifted to a position of $6.92 \mathrm{~mm}$ on $\mathrm{x}$-direction, $7.1 \mathrm{~mm}$ on y-direction and $0 \mathrm{~mm}$ on $\mathrm{z}$-direction, researchers got the desired triple band resonating frequencies. To attain an optimum performance, the patch has to be modified, and a rectangular slot with dimension of $37.2 \mathrm{~mm} \times 27.99 \mathrm{~mm}$ was initially drilled from the left side, in which the parameter was optimized to give a significant improvement of the antenna.

Design specifications. The three essential parameters for the design of a rectangular microstrip patch antenna are Frequency of operation ( $\mathrm{fr}$ ). The resonant frequency of the antenna must be selected appropriately. Wifi systems for communications use the frequency range from 2.4GHZ to $2.45 \mathrm{GHZ}$ [8]. Hence the antenna design must be able to operate in this frequency as solution frequency. The resonant frequency selected for this design is $2.45 \mathrm{GHZ}$. The second is Dielectric constant of the substrate $\left(\varepsilon_{r}\right)$. The dielectric material of this design has a dielectric constant of 4.4. Asubstrate with a high dielectric constant can reduce the dimensions of the antenna. However, for the radiation modes, most of them use such substrate result in elements, which are electrically small in terms of free space wavelengths and consequently have relatively smaller bandwidths and low efficiencies. The third is height of dielectric 
substrate (h). For the microstrip patch antenna to be used in communications applications and phones etc, it is essential that the antenna is not bulky. Hence, the height of the dielectric substrate selected here is $1.6 \mathrm{~mm}$. The essential parameters of the design are: $\mathrm{fr}=2.45 \mathrm{GHZ}, \varepsilon_{r}=4.4$, and $\mathrm{h}=1.6 \mathrm{~mm}$.

Design procedure. The design of a low profile, wide band multiband patch antenna is very complicated. The fact is that the lowest antenna profile, the widest impedance bandwidth and the most omni-directional (lowest ripple level) azimuth plane radiation pattern cannot be achieved simultaneously [9]-[11]. 5 as

Step 1. Calculation of the Width (W): The width of the microstrip patch antenna is given by equation

$$
\mathrm{W}=\frac{c}{2 f_{r}} \sqrt{\frac{2}{\left(\varepsilon_{r}+1\right)}}
$$

Substituting c $=3 \mathrm{e} 8 \mathrm{~m} / \mathrm{s}, \varepsilon_{r}=4.4$ and fr $=2.45 \mathrm{GHZ}$. We get $\mathrm{W}=37.2 \mathrm{~mm}$.

Step 2. Calculation of effective Dielectric Constant $\left(\varepsilon_{e f f}\right)$ : Equation 6 gives the effective dielectric constant as

$$
\varepsilon_{e f f}=\frac{\varepsilon_{r}+1}{2}+\frac{\varepsilon_{r}-1}{2}\left[1+12 \frac{h}{w}\right]^{-\frac{1}{2}}
$$

Substituting $\varepsilon_{r}=4.4, \mathrm{w}=37.2 \mathrm{~mm}$ and $\mathrm{h}=1.6 \mathrm{~mm}$ we get $\varepsilon_{\text {eff }}=4.08$.

Step 3. Calculation of the effective length ( $\mathrm{L}_{\mathrm{eff}}$ ) gives the effective length as

$$
\mathrm{L}_{\mathrm{eff}}=\frac{c}{2 f_{r} \sqrt{\varepsilon_{\text {eff }}}}
$$

Substituting $\varepsilon_{\text {eff }}=4.08, \mathrm{c}=3 \mathrm{e} 8 \mathrm{~m} / \mathrm{s}, \mathrm{fr}=2.45 \mathrm{GHZ}$, we get $\mathrm{L}_{\mathrm{eff}}=30.3 \mathrm{~mm}$.

Step 4. Calculation of the length extension $(\Delta \mathrm{L})$ : Give the length extension as

$$
\Delta \mathrm{L}=0.412 \mathrm{~h} \frac{\left(\varepsilon_{\text {eff }}+0.3\right)\left[\frac{w}{h}+0.264\right]}{\left(\varepsilon_{\text {eff }}-0.258\right)\left[\frac{w}{h}+0.8\right]}
$$

Substituting, $\varepsilon_{\text {eff }}=4.08, \mathrm{~W}=37.2 \mathrm{~mm}$ and $\mathrm{h}=1.6 \mathrm{~mm}$, we get $\Delta \mathrm{L}=0.74 \mathrm{~mm}$.

Step 5. Calculation of the actual length of the patch (L): The actual length is obtained by subtracting the length extension from the effective length as

$$
\mathrm{L}=\mathrm{L}_{\mathrm{eff}}-2 \Delta \mathrm{L}
$$

Substituting $\mathrm{L}_{\mathrm{eff}}=30.3 \mathrm{~mm}$ and $\Delta \mathrm{L}=0.74 \mathrm{~mm}$, researchers get $\mathrm{L}=28.82 \mathrm{~mm}$.

Step 6. Determination of feed point location (xf,yf): A coaxial probe type feed were used in the design, as shown in the Fig. 1, the centre of the patch is taken as the origin, and the feed point location is given by the coordinates (xf, yf) from the origin. The feed point must be located at that point on the patch, where the input impedance is $50 \mathrm{ohms}$ for resonant frequency.

As can be seen from the above calculations, it is found that the antenna size and the dimensions are large to be practically applied in wireless applications. Therefore, to achieve a smaller antenna size, researchers need to shorten the circuit radiating patch to the antenna ground plane with a shorting pin, and utilize an asymmetric branch line slit to meander the excited patch surface currents in the top patch, resulting in a large reduction of the required top patch dimensions. The ground plane dimension is modified to fit in to the PCB dimensions of most wireless applications like handsets

From Fig. 1, the Ls stands for the length of the substrate, Ws stands for the width of the substrate, Lp stands for the length of the patch while $\mathrm{Wp}$ is the width of the patch, the radius of the feeding port was 
$1.5 \mathrm{~mm}$, and the geometry is not drawn to a scale. In order to calculate the full three-dimensional electromagnetic field inside a structure and the corresponding S-parameters, HFSS software is used.

\section{Simulation Result}

The simulation result is summarized in Table 1, and the radiation pattern of the three bands is presented in $\mathrm{E}$ and $\mathrm{H}$ planes respectively, as shown in Fig. 2. The exercise endeavours to examine triple band frequencies of a microstrip patch antenna in which the frequency bands of $1.9 \mathrm{GHZ}, 2.45 \mathrm{GHZ}$ and 3.19GHZ was realized, also, the return loss and voltage standing wave ratio were also realized as expected.

Table 1 Simulation Result

\begin{tabular}{cccc}
\hline Frequency $(\mathbf{G H z})$ & Return Loss $(\mathbf{d B})$ & VSWR & Bandwidth $(\mathbf{G H z})$ \\
\hline 1.90 & -20 & 1.3 & 0.14 \\
2.45 & -36 & 1.06 & 0.14 \\
3.19 & -20 & 1.3 & 0.19 \\
\hline
\end{tabular}

The return loss (RL) is a parameter. It indicates the amount of power reflected from load. When wave reflects, leads to the standing wave, and antenna impedance does not match. Hence the RL is related to voltage standing wave ratio. In order to indicate how well the matching between the transmitter and the antenna, we use the RL and $\Gamma$. For perfect matching between the transmitter and the antenna, $\Gamma=0$ and $\mathrm{RL}=\equiv$. These conditions mean that no power reflected back. If $\Gamma=1$ and $\mathrm{RL}=0 \mathrm{~dB}$, it implies that all of incident power has been reflected, and the antenna can not work. The minimum VSWR corresponds to a perfect match, we have VSWR $=1$. Generally, the VSWR $>1$ since most of the radio equipment is not built for perfect match of the impedance. For our design above, the VSWR is 1.3 at $1.9 \mathrm{GHZ}, 1.06$ at 2.45GHZ, and 1.3 at 3.19GHZ respectively. Table 1 shows good performance of our designed antenna.

\section{Radiation Characteristics}

To examine the radiation of the composite triple band antenna structures, radiation pattern has been simulated; result is shown in Fig. 2. According to the far field radiation pattern $E_{\theta}$ at $\varphi=0^{\circ}$ and $90^{\circ}$ and frequency at the $1.9 \mathrm{GHZ}, 2.45 \mathrm{GHZ}$ and $3.19 \mathrm{GHZ}$ bands. Result shows that three radiation patterns are less than $10 \mathrm{~dB}$ level variation, which can be used for wireless applications appropriately.

Fig. 2(a) is the radiation pattern of the $1.9 \mathrm{GHZ}$ frequency, Fig. 2(b) and (c) are also of the $2.45 \mathrm{GHZ}$ and 3.19GHZ frequencies. All results have been obtained by using the high frequency structured simulator. In order to obtain a more clear picture of the radiation pattern of the antenna, we use the origin software after the simulation of the antenna by high frequency structure simulator, as shown in the above figures.

\section{Conclusion}

This paper has made use of HFSS software, in which a triple bands microstrip patch antenna has been designed and simulated. From the result of the simulation, it has been observed that the influencing parameters of the antenna are the relative permittivity of the dielectric under the patch, feed location, the position of the parasitic patch, the length and width of the patch and finally the thickness of the substrate. 
Triple bands frequency of 1.9GHZ,2.45GHZ and 3.19GHZ has been realized with their corresponding return loss of $-20 \mathrm{~dB},-36 \mathrm{~dB}$ and $-20 \mathrm{~dB}$ as well as their respective voltage standing wave ratios of 1.3 , 1.06 and 1.3 .

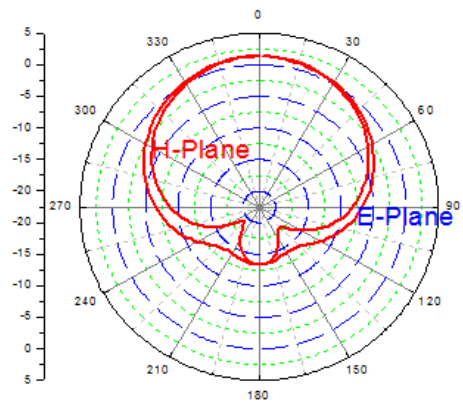

(a)

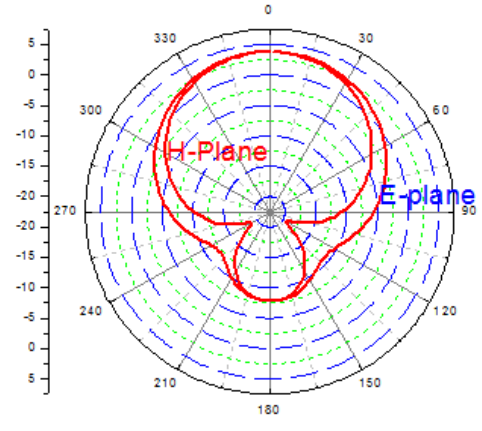

(b)

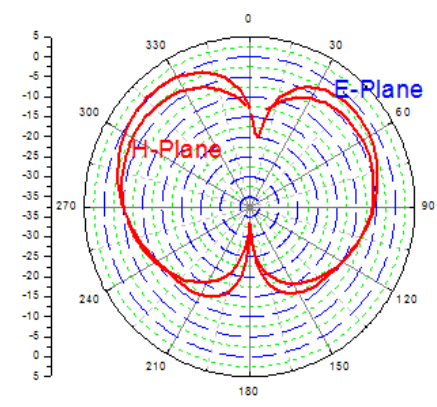

(c)

Fig. 2 Radiation Pattern at (a) $1.9 \mathrm{GHz}$, (b) $2.45 \mathrm{GHz}$, and (c) $3.19 \mathrm{GHz}$.

\section{Acknowledgments}

This work was supported by the National Nature Science Foundation of China, the Natural Science Foundation of Tianjin Municipality and Advanced Technology, China, and the Science Research and Development Foundation of Tianjin University of Technology and Education, China, Grant 61371043, 12JCYBJC10500, and KJY14-04, respectively.

\section{References}

[1] C. A. Balanis, Antenna Theory Analysis and Design, 2nd edition, John Wiley \& sons, Inc, 2005.

[2] G. Sami, M. Mohanna, M. L. Rabeh, Tri-band microstrip Antenna Design for Wireless Communication Applications, Journal of Astronomy and Geophysics, 2(2013) 39-44.

[3] Seddik Br, S. Zaakri, A. Nakheli, and A. Mamouni, Simulations of Dual and Broadband Patch Antenna, European Journal of Scientific Research, 60(2011) 237-249.

[4] M. A. S. Alkanhal, Composite Compact Triple-band Microstrip Antennas, Progress In Electromagnetic Research, PIER 93(2009) 221-236.

[5] J. D. Kraus, Antennas, 2nd Edition, McGaraw-Hill company, 1988.

[6] S. N. Makarov, Antenna and EM Modelling with MATLAB, John Wiley \& Sons, Inc., 2002.

[7] Agilent High Frequency Structure Simulator User Guide, 2000.

[8] C. A. Felippa, Introduction to Finite Element Method, Available online at http://www.colorado. edu/courses.d/IFEM.ch01.pdf.

[9] W. L. Stutzman, G. A. Thiele, Antenna Theory and Design, John wiley \& Sons, 1997.

[10]M. Iftisane, S. Bri, L. Bellarbi, Conception of Patch Antenna in the GSM and UMTS Band. Progress in Electromagnetic Research Symposium, Xian, China, (2010) 1150-1155.

[11]K.-L. Wong, Compact and Broadband Microstrip Antenna, Wiley Series in Microwave and Optical Engineering, (2002) 279-294. 